\title{
Immunohistochemical Characterization of Cell of Origin in Diffuse Large B-cell Lymphoma and its Correlation with the Double Expressor Phenotype: A Retrospective Study in a Tertiary Cancer Care Centre
}

\section{suparno Chakrabarti}

Dharamshila Hospital and Research Centre: Dharamshila Narayana Superspeciality Hospital Hema MALINI aiyer

Dharamshila Hospital and Research Centre: Dharamshila Narayana Superspeciality Hospital gaurav sharma

Dharamshila Hospital and Research Centre: Dharamshila Narayana Superspeciality Hospital sarita rani jaiswal

Dharamshila Cancer Hospital: Dharamshila Narayana Superspeciality Hospital

Prachi prachi ( $\nabla$ prachipath123@gmail.com)

Dharamshila Hospital and Research Centre: Dharamshila Narayana Superspeciality Hospital https://orcid.org/0000-0002-6069-5559

\section{Research}

Keywords: Cell-of-origin, Immunohistochemistry, Hans and Choi algorithm, Double Expressor.

Posted Date: August 18th, 2021

DOI: https://doi.org/10.21203/rs.3.rs-763051/v1

License: (c) (i) This work is licensed under a Creative Commons Attribution 4.0 International License. Read Full License 


\section{Abstract}

Background - Diffuse Large B Cell Lymphoma (DLBCL) is the most common type of Non- Hodgkin Lymphoma (NHL) and is categorized into the Germinal Centre B-cell (GCB) and Activated B-cell (ABC/ Non-GCB) subtypes as per the Cell-of-origin ( $\mathrm{COO}$ ) model with the help of Gene Expression Profiling/ Immunohistochemistry. The non -GCB subtype has been found to correlate with the Double Expressor (DE) phenotype (i.e. co-expression of bcl-2 and c-myc by IHC) and this correlation was substantiated and proven to be statistically significant in the present study.

Results- $A$ retrospective study of 50 patients was carried out with the help of archival material filed in the Department of Anatomic Pathology at Dharamshila Narayana Superspeciality Hospital from $1^{\text {st }}$ January 2019 to $30^{\text {th }}$ June 2020. The study cohort was divided into two Groups - Group-A with nodal presentation and Group -B with extranodal presentation. By using the Hans and Choi IHC algorithms, the cases were categorized into the GCB and non - GCB subtypes in both the groups. The DE phenotype was determined in each case by the co-expression of c-myc (>40\%) and bcl-2 (>50\%) by IHC.

Conclusion - Due to the heterogeneity inherent in DLBCL, prediction of the Double Expressor phenotype and the $\mathrm{COO}$ by $\mathrm{IHC}$ is a sensitive tool and helps in the prognostication and therapeutic triage of patients. Hence in all cases diagnosed as DLBCL, a detailed morphological and IHC work up is mandatory to determine prognosis and possibly tailor therapy according to cell of origin and double expressor phenotype.

\section{Abstract Table}

Correlation of DE phenotype in the GCB and non-GCB subtypes

\begin{tabular}{|c|c|c|c|}
\hline \multicolumn{3}{|c|}{ Total patients ( 50 ) } & \\
\hline \multicolumn{2}{|c|}{ GCB (12 cases) } & Non-GCB( 38 cases) & \\
\hline DE phenotype & $1(6.7 \%)$ & $25(66 \%)$ & $\begin{array}{l}\text { Chi-square Value }=12.062 \\
\mathrm{Df}=1 \\
\text { P value }=0.0005 \\
\text { Yates Correction }=9.87 \\
P \text { value }=0.00168 \\
\text { Significant }\end{array}$ \\
\hline
\end{tabular}

\section{Background}

DLBCL is the most common type of aggressive Non Hodgkin Lymphoma, representing approximately $24 \%$ of all newly diagnosed cases of NHL. It represents a heterogenous group of diseases with variable outcomes that are differentially characterized by clinical features and most recently by recurring mutations. ${ }^{[1,2]}$ 
Clinically patients present with rapidly enlarging lymphadenopathy, along with constitutional symptoms. There is a high frequency of extranodal disease, if there is generalized lymphadenopathy.

The "cell of origin" model divides DLBCL into the Germinal centre B-cell (GCB) type and Non Germinal centre B-cell type or Activated B- cell (ABC) type based on the pattern of gene expression. The gene expression of the Germinal Centre type fits with the normal germinal centre derived $B$ cell and in the subtype non -GCB DLBCL or activated B-cell lymphoma, the gene expression profiling more closely fits in with a normal activated B-cell.

"Cell of origin" (COO) model also determines the prognosis of the two biological subtypes of DLBCL, that should be treated differently.

C-myc is a proto-oncogene on chromosome $8 \mathrm{q} 24$ and encodes a transcription factor, which when dysregulated leads to cellular survival and proliferation. Bcl-2 is an oncogene with an anti-apoptotic property.

Myc rearrangement $\mathrm{t}(8 ; 14)$ is prototypically associated with Burkitt lymphoma but is also associated with 12 to $15 \%$ of DLBCL. BCL-2 rearrangement $t(14 ; 18)$ is also important as it leads to a drug resistant phenotype with increase in cancer cell survival.

Thus a concurrent rearrangement of $\mathrm{Bcl}-2$ and c-myc, which are present in approximately 5 to $7 \%$ of DLBCL, leads to a clinically resistant formof DLBCL, termed as a Double Hit Lymphoma and is associated with a poor prognosis. ${ }^{[3]}$

The revised WHO classification recognizes the co-expression of myc (>40\%) and bcl-2 (>50\%) proteins as a new adverse prognostic marker within DLBCL, NOS as " Double Expressor Lymphoma”. ${ }^{4,5,6]}$

Another variant of the double hit lymphoma presents with co-rearrangement of c-myc and bcl-6 genes.

All the 3 genes bcl-2, myc and bcl-6 are simultaneously rearranged in the phenotype termed as "Triple -Hit Lymphoma".

Patient who have the double hit rearrangement usually have protein over- expression and therefore have the double expressor phenotype. However the converse is not always true. ${ }^{[7]}$

Double hit lymphoma occurs more commonly in GCB type of DLBCL, while DE Lymphoma is commoner in the Non-GCB type of DLBCL.

Double expressors are detected by IHC staining and Double Hit Lymphomas are detected by FISH.

The most common upfront treatment for Double Expressor Lymphomas is chemoimmunotherapy (Cl) with R-CHOP (Rituximab, Cyclophosphamide, Doxorubicin, Vincristine and Prednisolone) which leads to a cure in $50-60 \%$ of patients. Patients who develop disease that is refractory to upfront treatment or 
relapse after achieving remission, are treated with autologous stem cell transplantation (ASCT) and they generally have a poor outcome.

The majority of patients with Double Expressor Lymphoma relapse after R-CHOP${ }^{[8]}$, hence such patients should be treated with ASCT. For patients unable to undergo transplantation, the median survival is 6 months.

CART (Chimeric Antigen Receptor T-cell Therapy) is promising for patients with aggressive B-Cell Lymphomas, that do not respond to other treatment modalities.

Therefore all patients of DLBCL should be assessed for the DE phenotype, which aids in risk stratification of the patient and further helps in optimizing the treatment strategy.

\section{Methods}

This was a retrospective study to validate a model to predict the DE phenotype based on COO subtype. All newly diagnosed DLBCL patients were divided into Group A including patients with Nodal involvement and Group B including patients with Extranodal involvement.

Based on "COO" both the groups were further divided into GCB type and Non GCB type of DLBCL. With the help of $\mathrm{IHC}$ using the Hans or Choi algorithm IHC staining for bcl-2 \& c-myc was used to determine the DE phenotype and correlated with the COO subtypes of DLBCL.

Our study includes 50 patients, assessed over a period of one and half years from 1 st January 2019 to 30th June 2020, who presented with generalized lymphadenopathy and/or Extranodal disease along with other associated constitutional symptoms.

A Biopsy was performed in each case and sent in $10 \%$ neutral buffered formalin to the Department of Surgical Pathology for further processing and evaluation. Formalin fixed paraffin embedded tissue sections were cut and mounted on slides and stained with routine Haematoxylin and Eosin stain.

Further, IHC stains were applied to assess the 'COO' and DE phenotypes based on the percentage of staining of the cells and were correlated.

In all cases Immunohistochemistry was performed using the automated Roche Ventana Benchmark XT IHC Autostainer (Ventana Medical Systems Inc. Tucson, Arizona) with the aid of the UltraView Universal DAB Detection Kit.

The antibodies and clones employed were- CD3 [PS-1], CD5 [4C-7], CD10 [56C6], CD20 [L-26], CD30 [BerH2], Bcl-2 [EP-36], Bcl-6[EP278], C-myc [EP121], MUM-1 [EP190], Ki-67[30 - 9] and FOXP-1[EP137] provided by PATHNSITU BIOTECHNOLOGIES PVT LTD and GCET-1[ab6888] from ABCAM (Cambridge, U.K.) [Figure 1-10] 
Co expression of c-myc $(>/=40 \%)$ and bcl-2(> 50\%) in neoplastic cells by IHC staining was considered as DE phenotype.

Ethics approval and Consent to participate- The study was approved by Instituitional Ethics Committee, (Name of committee-Dharamshila Narayana Superspeciality Hospital) vide registration no. $\mathrm{ECR} / 226 /$ Inst/DL/2019.

\section{Results}

The present study includes 50 patients, who presented to our institution during a period of one and a half years from the $1^{\text {st }}$ January 2019 to $30^{\text {th }}$ June 2020. The median age at presentation was 49 years (20-81 years) with a male to female ratio of 2:1. Group A with nodal disease includes 28 patients and Group B with Extranodal disease includes 22 patients, constituting $56 \%$ and $44 \%$ respectively of the study cohort.

Histological sections examined from biopsy specimens showed diffuse infiltration by predominantly large atypical lymphoid cells with prominent nucleoli and mitotic activity. All cases were positive for the pan B-Cell marker CD 20 and a few showed positivity for CD30.

8 of the 28 patients in Group A were categorized as GCB type and 20 (71.4\%) were categorized as non GCB type by immunohistochemistry, using the Hans and Choi algorithm. Out of the 8 GCB type, only 1 (12.5\%) had a Double Expressor phenotype, while 7 (87.5\%) were non Double expressor type. Out of the 20 non GCB type, 15 (75\%) had a DE phenotype and 5 (25\%) were of the non DE type.

4 of the 22 patients in Group B (18\%) were categorized as GCB type and $18(81.9 \%)$ as non -GCB type. Out of the 4 GCB type, none showed a DE phenotype. However, 10 of the 18 non GCB type (55.6\%) were DE type and 8 (44.4\%) were non DE type.

The DE phenotype was present in $26 / 50$ (52\%) and the NDE phenotype was expressed in $24 / 50(48 \%)$ of cases included in the present study.

While in the non GCB type DE phenotype was expressed in 25 cases (65.8\%) out of 38 cases, only 1 of the 12 GCB cases expressed the DE phenotype (8.3\%). [Refer table $1,2,3]$

Table 1: Incidence of DE phenotype in the GCB and non GCB subtype in Group A 


\begin{tabular}{|c|c|c|c|}
\hline \multicolumn{4}{|c|}{ Nodal Group A (28 patients ) } \\
\hline \multicolumn{2}{|c|}{ GCB (8 cases) } & \multicolumn{2}{|l|}{ Non GCB (ABC) (20 cases) } \\
\hline \multirow[t]{3}{*}{ DE phenotype } & $1(12.5 \%)$ & $15(75 \%)^{\star}$ & Chi-square Value $=9.115$ \\
\hline & & & $D f=1$ \\
\hline & & & $P$ value $=0.002$ \\
\hline \multirow[t]{3}{*}{ NDE phenotype } & $7(87.5 \%)$ & $5(25 \%)$ & Yates Correction=6.741 \\
\hline & & & $P$ value $=0.009$ \\
\hline & & & Significant \\
\hline
\end{tabular}

*value in parenthesis shows percentage

Thus in the Nodal Group the Non- GCB subtype correlated with the Double expressor Phenotype and the association was found to be statistically significant $(p-0.009)$

Table 2: Incidence of DE phenotype in the GCB and non GCB subtypes in Group B

\begin{tabular}{|llll|}
\hline \multicolumn{3}{|c|}{ Extra -Nodal Group B (22 patients) } \\
\hline GCB (4 cases) & Non GCB (18cases) \\
\hline DE phenotype & $0(0.0)$ & $10(55.6 \%)^{*}$ & Chi-square Value $=4.074$ \\
& & & Df=1 \\
& & P value $=0.04$ \\
NDE phenotype & $4(100 \%)$ & $8(44.4 \%)$ & Yates Correction $=2.141$ \\
& & & P value $=0.1434$ \\
& & & Nonsignificant \\
\hline
\end{tabular}

*value in parenthesis shows percentage

In the Extranodal group though the non GCB subtype showed the DE phenotype more commonly than the NDE phenotype, the association was not found to be statistically significant (p-0.1434).

Table 3: Overall Incidence of DE phenotype in the GCB and non GCBsubtype 


\begin{tabular}{|c|c|c|c|}
\hline \multicolumn{4}{|c|}{ Total patients $(50)$} \\
\hline \multicolumn{2}{|c|}{ GCB (12 cases) } & \multicolumn{2}{|l|}{ Non GCB(38 cases) } \\
\hline \multirow[t]{3}{*}{ DE phenotype } & $1(6.7 \%)$ & $25(66 \%)^{*}$ & Chi-square Value $=12.062$ \\
\hline & & & $D f=1$ \\
\hline & & & $P$ value $=0.0005$ \\
\hline \multirow[t]{3}{*}{ NDE } & $11(93.3 \%)$ & $13(34 \%)$ & Yates Correction $=9.87$ \\
\hline & & & $P$ value $=0.00168$ \\
\hline & & & Significant \\
\hline
\end{tabular}

*value in parenthesis shows percentage

Overall the Double Expressor phenotype correlated with the non- GCB Subtype and the NDE phenotype with the GCB subtype. The association was found to be statistically significant.

Thus the DE phenotype correlated with the non- GCB subtype and the NDE phenotype with the GCB subtype in this study

\section{Discussion}

Cell of Origin - COO

According to the 2016 WHO classification, diagnosis of all cases of DLBCL, NOS should include COO, (GCB vs. ABC or non-GCB, if an IHC algorithm is used), because of their different molecular features, biologic behavior, prognosis and treatment.

The starting point in a discussion about prognostic variables in Diffuse Large B-cell lymphoma is the observation that some patients can be cured and others cannot. Several studies have investigated the biologic underpinnings for these different outcomes. A pioneering genetic evaluation of diffuse large Bcell lymphoma (DLBCL), published by Alizadeh and colleagues in 2000, showed 2 subtypes: germinal center and non-germinal center (also known as activated $B$-cell/ABC).

This distinction is important because better outcomes are seen in patients with the germinal-center subtype than with the non-germinal-center subtype. We therefore now have a "cell-of-origin" model showing that there are at least 2 genetic biologic types of DLBCL that might explain prognosis and could perhaps be targeted differently. This insight has led to recent trials that test new treatments in one genetic subtype vs the other. We are hopefully very close to understanding whether these 2 subtypes of DLBCL should be treated differently. ${ }^{[15]}$ 
The most challenging profile to detect in routine practice is "cell of origin." The gold standard for identifying germinal-centre DLBCL vs. non-germinal-centre DLBCL was first defined as gene-expression profiling patterns in frozen tumor material. However, gene-expression profiling is not routinely available nor is it considered a standard test. The most common approach is immunohistochemistry using different algorithms, such as the Hans, Choi or Tally algorithms, ${ }^{[9,10]}$ to determine whether a lymphoma is germinal-centre DLBCL or non-germinal-centre DLBCL. Compared with the gold standard of gene expression profiling in frozen material, there is an error rate of approximately $20 \%$ with immunohistochemical algorithms shown below.

The present study has used the Hans/Choi Algorithms to determine the Cell of Origin. ${ }^{[9,10]}$

Double-expressor lymphoma

The presence of both the $M Y C$ and $B C L 2$ rearrangements defines double-hit lymphoma (DHL). This phenotype is very proliferative and drug-resistant, and it is associated with a poor prognosis. Another variant of double-hit lymphoma is co-rearrangement of $M Y C$ and the BCL6 gene. Rarely, all 3 genes$B C L 2, M Y C$, and BCL6-are simultaneously rearranged in a phenotype termed triple-hit lymphoma (THL). Both double-hit and triple-hit lymphomas have a poor prognosis with standard treatment. ${ }^{[6,7]}$

Immunohistochemical staining to identify protein expression of $M Y C$ also showed that there are lymphomas in which $M Y C$ and $B C L 2$ genes are overexpressed at a protein level, without the genetic rearrangements. This profile has been referred to as the "double-expressor" phenotype in DLBCL in the revised 2016 World Health Organization (WHO) classification of lymphoid neoplasms. The WHO classification defines overexpression as greater than $40 \%$ c-MYC-expressing cells and greater than $50 \%$ BCL2-expressing cells by IHC. As shown in a study by Hu and colleagues, patients with double-expressor DLBCL have worse outcomes than patients in whom these proteins are not overexpressed; in general, only one-third of patients have long-term disease control with R-CHOP. ${ }^{[16]}$

Double-hit lymphoma is relatively uncommon, occurring in approximately $5 \%$ to $7 \%$ of patients with DLBCL. However Dual-expressor lymphomas may be present in as many as one-third of patients with DLBCL and serve to identify a significant subset of cases with a worse prognosis.

Patients who have the double-hit rearrangement usually have protein overexpression, and therefore have the double-expressor phenotype. However, the converse is not always true: dual-expressor protein overexpression is not always associated with an underlying double-hit rearrangement. Complicating the picture is that most double-hit lymphomas occur in the setting of a germinal-centre DLBCL, whereas most double-expressor lymphomas occur in non-germinal-centre DLBCL as demonstrated in this study which shows a strong correlation between the Double Expressor phenotype and the Non-GCB Cell of Origin subtype by IHC.

The double-hit lymphomas can be detected with fluorescence in situ hybridization (FISH) or standard cytogenetic analysis. The double-expressor lymphomas are diagnosed by immunohistochemistry. 
The double-expresser phenotype was not given a unique category, but was recognized by the WHO as a poor prognostic sign within DLBCL.

When patients with aggressive B-cell lymphomas relapse or become refractory to therapy, standard options are limited. For patients unable to undergo transplant, or for those who relapse after a stem cell transplant, the median survival is approximately 6 months. Despite the many trials that have tried to improve upon this dismal statistic, there are no breakthroughs at this time. Chimeric antigen receptor (CAR) T-cell therapy is exciting. This treatment is still in the early phases of research and associated with toxicity, but it is promising for patients with aggressive B-cell lymphomas that do not respond to other therapies. There are also a number of new biologic and targeted agents that are promising, and finding which patients may respond to a particular treatment is a high priority. ${ }^{[17]}$

\section{Conclusion}

According to the 2016 WHO classification, the diagnosis of DLBCL, NOS requires the inclusion of the COO (GCB or ABC/non-GCB subtype) determined either with molecular techniques (GEP and mRNA based techniques) or immunohistochemistry, as an alternative solution. The distinction of GCB versus ABCDLBCL has not yet led to differences in primary treatment. The current standard of care for most patients is R-CHOP, which has improved dramatically the outcome of DLBCL. However, for patients who fails to respond to R-CHOP, the choice of therapy is very likely to be influenced by the $\mathrm{COO}$ and the molecular pathways used by the tumors for survival and proliferation. Emerging new targeted therapy will certainly influence the diagnosis and treatment of DLBCL and HGBL in the near future. The routine use of FISH and $\mathrm{IHC}$ to detect $M Y C$ and $B C L 2$ alterations/overexpression is recommended.

Patients with DHL and double expression of MYC and BCL2 protein, represent poor-risk subsets in which alternative strategies should be explored.

HGBL with $M Y C$ and $B C L 2$ and/or BCL6 rearrangements (i.e., DHL or THL) should be separated from DLBCL, NOS, due to its clinicopathological features, molecular findings, and dismal prognosis with standard R-CHOP therapy.

Although there are no strict recommendations on how to select cases for FISH analysis, a reasonable approach is to perform FISH analysis for MYC, BCL2 and/or BCL6 in cases with aggressive clinical presentation, GCB phenotype, and double expression of MYC and BCL2 which has been shown to correlate with the Non-GCB Subtype as demonstrated by this study, thereby mandating a comprehensive and detailed morphologic and IHC work-up in all cases of High Grade B-cell Lymphoma in order to determine prognostic and therapeutically relevant and biologically distinct phenotypes .

\section{Abbreviations}

COO- cell of origin 
DE- double expressor

NDE-non double expressor

GCB- germinal centre B- cell

$A B C$ - activated B- cell

\section{Declarations}

Ethics and Consent to participate- The study was approved by Instituitional Ethics Committee,(Name of committee-Dharamshila Narayana Superspeciality Hospital) vide registration no.

ECR/226/Inst/DL/2019.

Consent for publication- written consent has been obtained

Competing interests- N/A

Funding- nil

Authors' contributions-

CONCEPT OF STUDY - SC, HMA,P

DEFINITION OF INTELLECTUAL CONTENT- SC, HMA,GS,SRJ,P

LITERATURE SEARCH - SC, HMA,GS,SRJ,P

CLINICAL/EXPERIMENTAL STUDIES- SC, HMA,GS,SRJ,P

DATA ACQUISITION - SC, HMA,GS,SRJ,P

DATA ANALYSIS- SC, HMA,GS,SRJ,P

MANUSCRIPT PREPARATION - SC, HMA,GS,SRJ,P

MANUSCRIPT EDITING - SC, HMA,GS,SRJ,P

MANUSCRIPT REVIEW - SC, HMA,GS,SRJ,P

All authors have read and approve the manuscript .

\section{Acknowledgement -}

The authors thank the entire technical staff, Suresh Chand Sharma, Barfi Ram Chaudhary and Nidhi Chaudhary from the Department of Pathology and entire technical team of Department of Haematooncology and Bone marrow transplantation, Dharamshila Narayana Superspeciality Hospital, New Delhi . 


\section{References}

1. SEER. SEER Database: All Lymphoid Neoplasms with Detailed Non- Hodgkin Lymphoma Subtypes; SEER b Incidence Rates and Annual Per- cent Change by Age at Diagnosis. NCl; 2002-2011.

2. Siegel R, Miller K, Jemal A. Cancer statistics, 2019. CA Cancer J Clin. 2019;69(1):7-34.

3. Swerdlow SH, Campo E, Pileri SA, et al: The 2016 revision of the World Health Organization classification of lymphoid neoplasms. Blood 127:2375-2390, 2016

4. Green TM, Young KH, Visco C, et al: Immunohistochemical double-hit score is a strong predictor of outcome in patients with diffuse large B-cell lymphoma treated with rituximab plus cyclophosphamide, doxorubicin, vincristine, and prednisone. J Clin Oncol 30:3460-3467, 2012

5. Horn $\mathrm{H}$, Ziepert M, Becher $\mathrm{C}$, et al: MYC status in concert with BCL2 and BCL6 expression predicts outcome in diffuse large B-cell lymphoma. Blood 121: 2253-2263, 2013

6. Johnson NA, Slack GW, Savage KJ, et al: Concurrent expression of MYC and BCL2 in diffuse large Bcell lymphoma treated with rituximab plus cyclophosphamide, doxorubicin, vincristine, and prednisone. J Clin Oncol 30:3452-3459, 2012

7. Johnson NA, Savage KJ, Ludkovski O, et al: Lymphomas with concurrent BCL2 and MYC translocations: The critical factors associated with survival. Blood 114:2273-2279, 2009

8. Herrera AF, Mei M, Low L, et al: Relapsed or refractory double-expressor and double-hit lymphomas have inferior progression-free survival after autologous stem-cell transplantation. J Clin Oncol 35:2431,2017

9. Hans CP, Weisenburger DD, Greiner TC, et al: Confirmation of the molecular classification of diffuse large B-Cell lymphoma by immunohistochemistry using a tissue microarray.Blood.2004; 103(1):275282.

10. Choi WWL, Weisenburger DD, Greiner TC, et al: A new immunostain algorithm classifies diffuse large B -cell lymphoma into molecular subtypes with high accuracy. Clin cancer res.2009; 15:5494-502.

11. C Visco, Y Li, Z Y Xu-Monette, R N Miranda, T M Green, A Tzankov, et al: Comprehensive gene expression profiling and immunohistochemical studies support application of immunophenotypic algorithm for molecular subtype classification in diffuse large B-cell lymphoma: a report from the International DLBCL Rituximab -CHOP Consortium Program Study. Leukemia.2012 ;26:2103-113.

12. Gutiérrez-García, G, Cardesa-Salzmann, T, Climent, F, et al: Gene- expression profiling and not immunophenotypic algorithms predicts prognosis in patients with diffuse large B-cell lymphoma treated with immunochemotherapy. Blood. 2011;117(18):4836-4843.

13. Scott DW, King RL, Staiger AM, et al. High grade B-cell lymphoma with MYC and BCL2 and/ or BCL6 rearrangements with diffuse large B-cell lymphoma morphology. Blood. 2018;131(18):2060-2064.

14. Ennishi D, Jiang A, Boyle M, et al. Double-hit gene expression signature defines a distinct subgroup of germinal centre B-cell-like diffuse large B-cell lymphoma. J Clin Oncol. 2019;37(3):190-201. 
15. Alizadeh AA, Eisen MB, Davis RE, et al. Distinct type of diffuse large B-cell lymphoma identified by gene expression profiling. Nature.2000; 403 (6769): 503-511.

16. Hu S, Xu Monette ZY, Balasubramanyam A, et al. CD30 expression defines a novel subgroup of diffuse large - B-cell Lymphoma with favourable prognosis and distinct gene expression signature: a report from the International DLBCL Rituximab -CHOP Consortium Program Study. Blood.2013;121:2715-2724.

17. Schuster S, Bishop M, Tam S, et al. Tisagenlecleucel in adult relapsed or refractory diffuse large Bcell Lymphoma . N Engl J Med.2019; 380: 45-56.

\section{Figures}

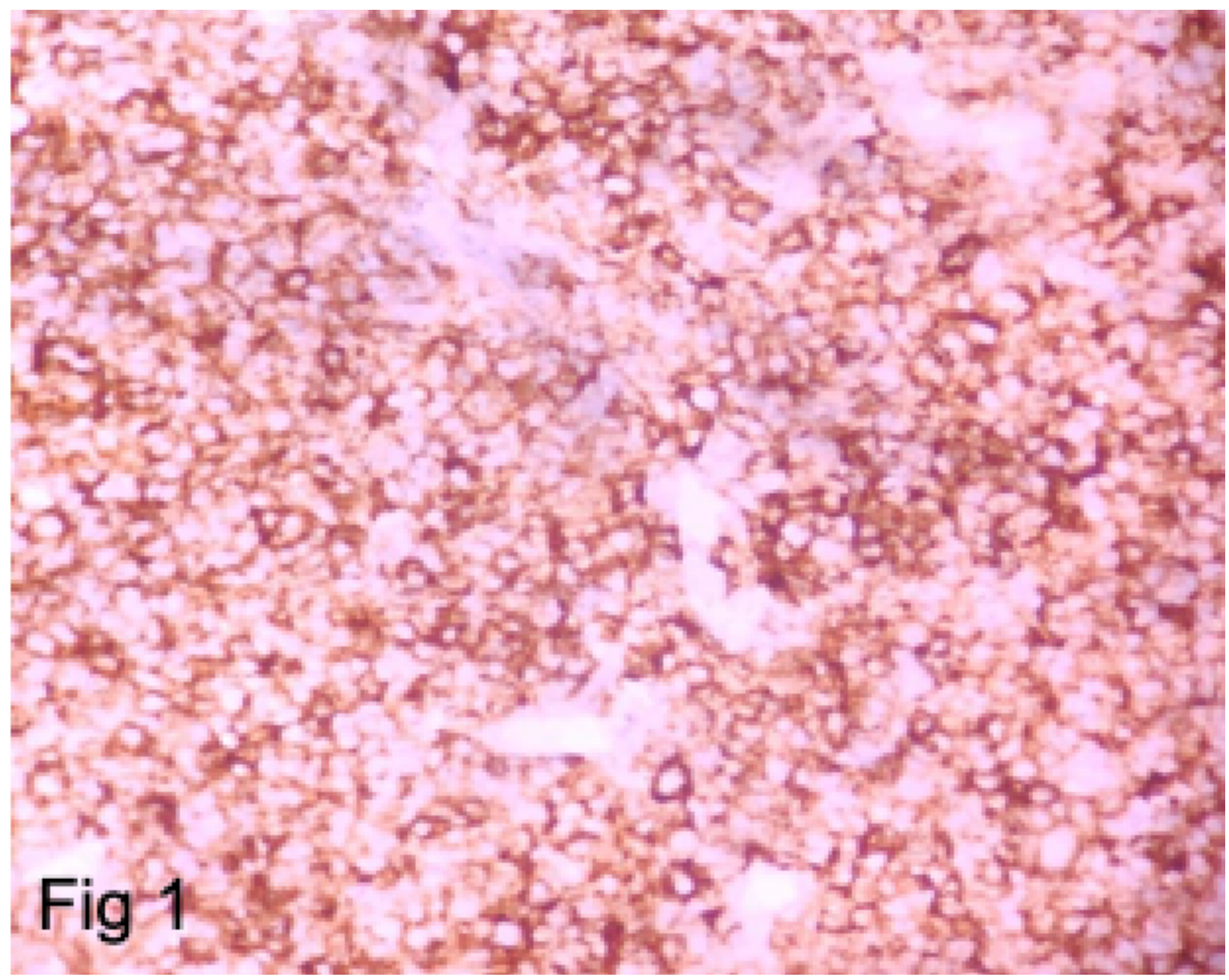

\section{Figure 1}

Diffuse strong membrane staining for CD20 in Lymphoma cells ( $x 400)$ 


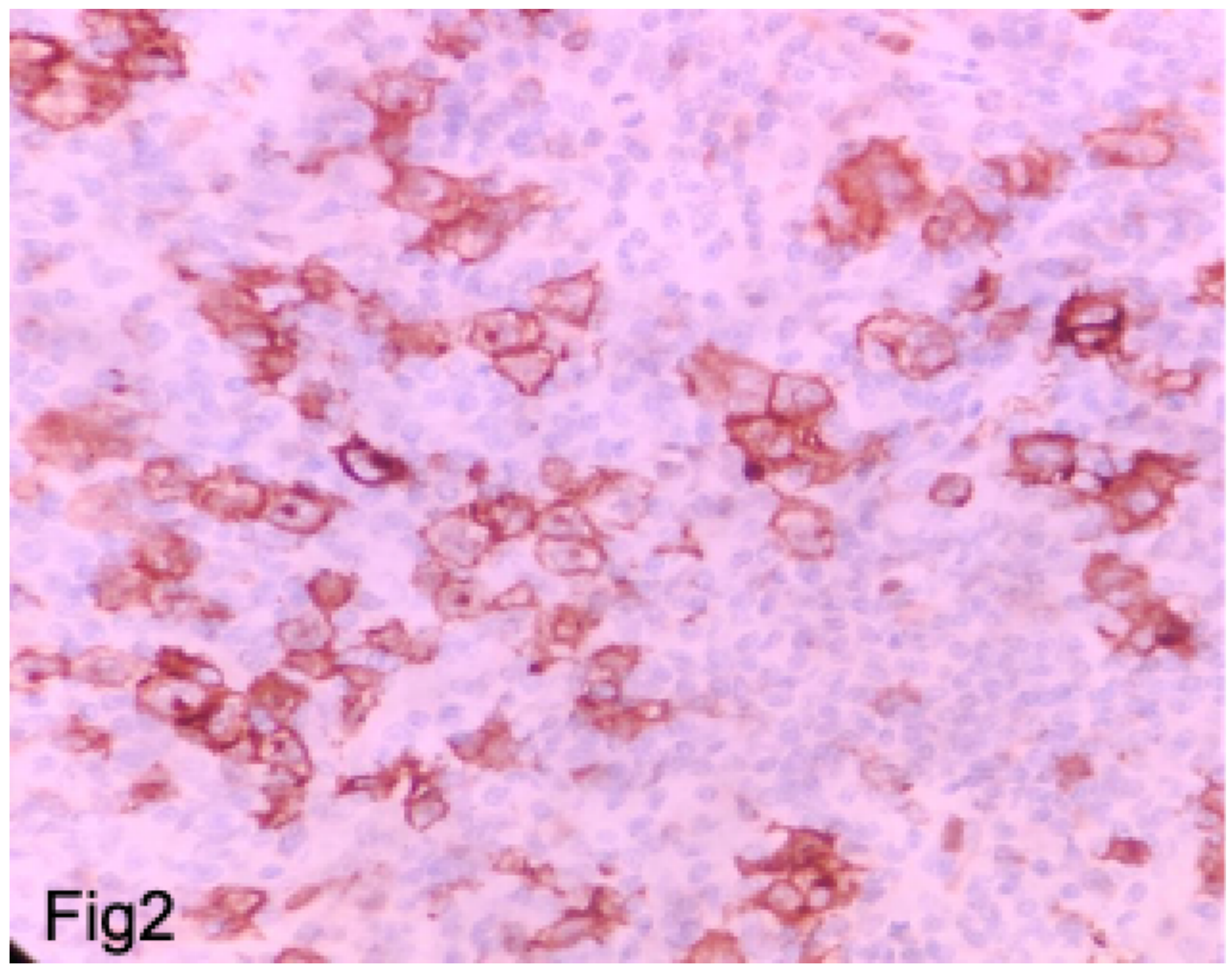

Figure 2

Patchy membrane and golgi zone staining for CD30 seen in some cases $(x 400)$ 


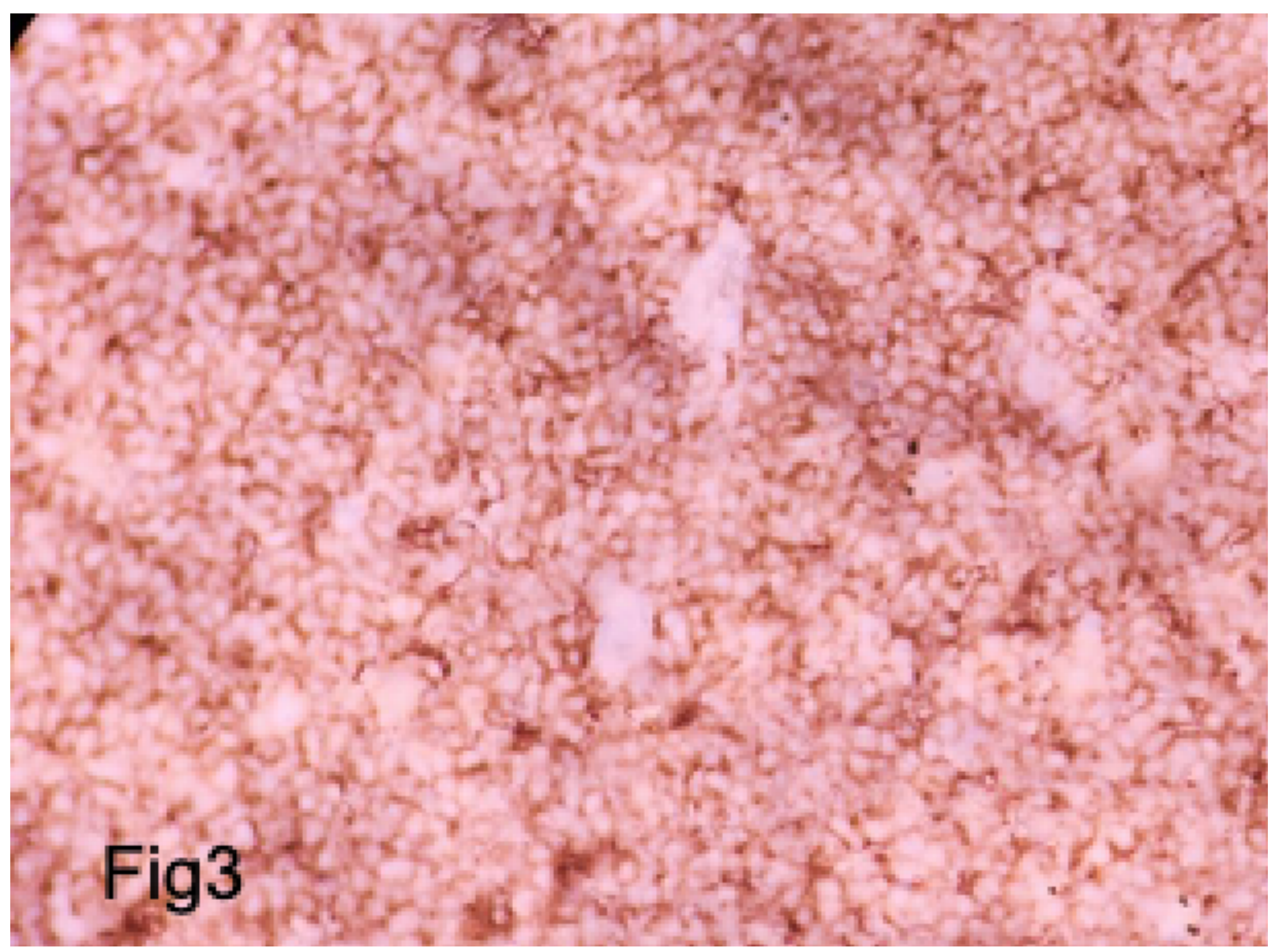

Figure 3

Diffuse membrane staining for CD10 in tumor cells in DLBCL,GCB type (x400) 


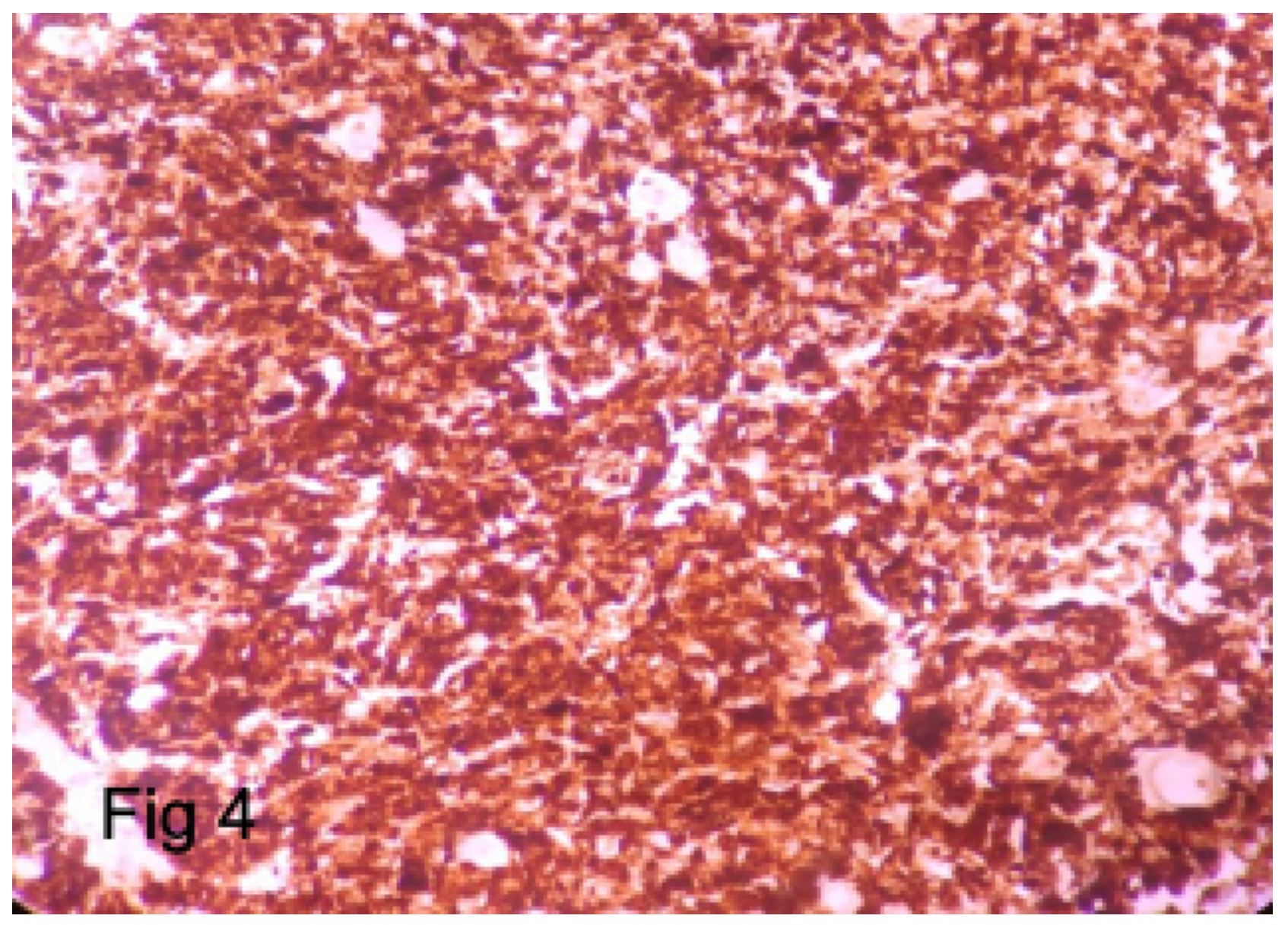

Figure 4

Diffuse strong granular cytoplasmic staining for GCET-1 in DLBCL,GCB type ( $x 400$ ) 


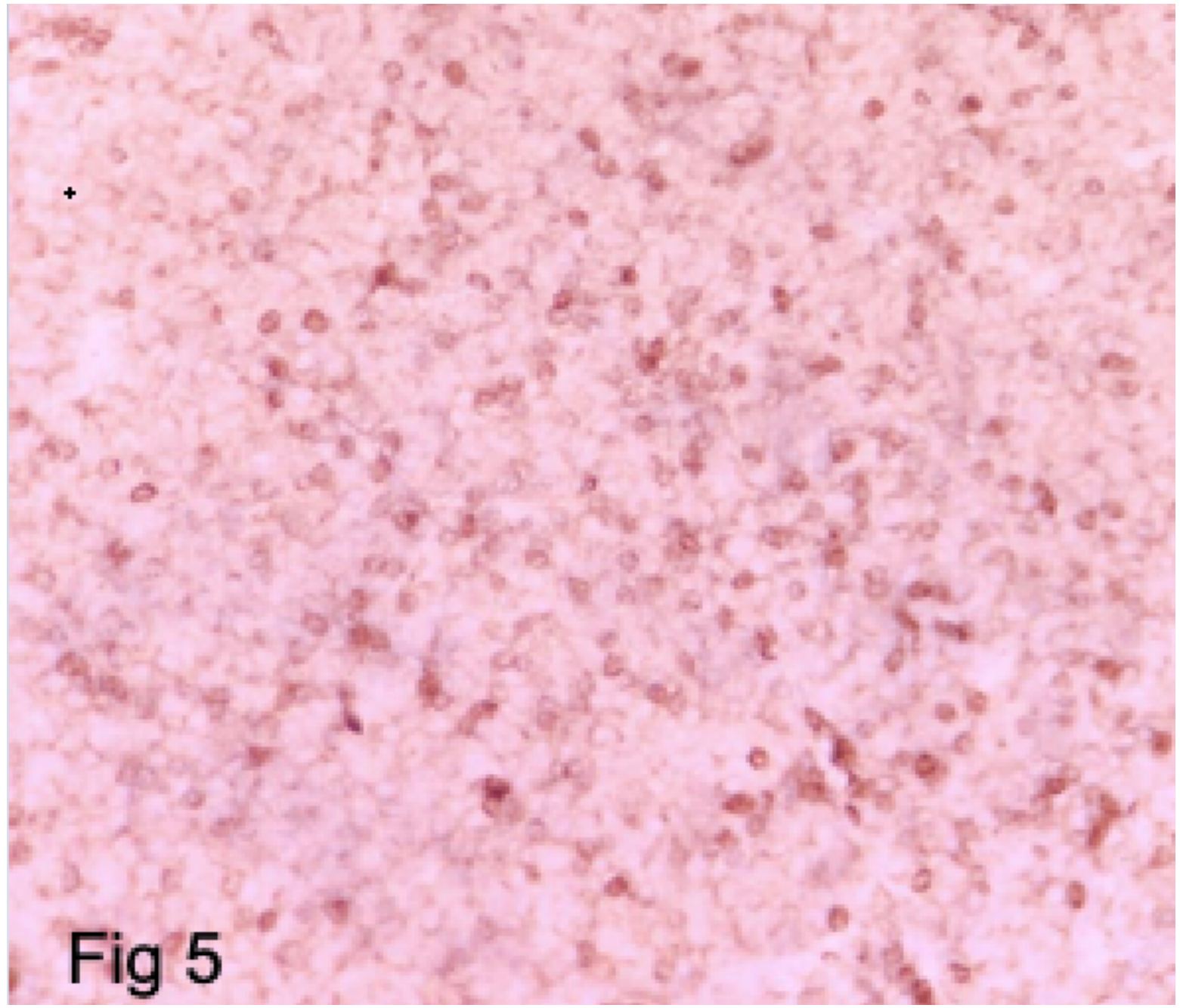

Figure 5

Diffuse staining for bcl-2 in $>50 \%$ lymphoma cells in Double expressor type DLBCL (X400) 


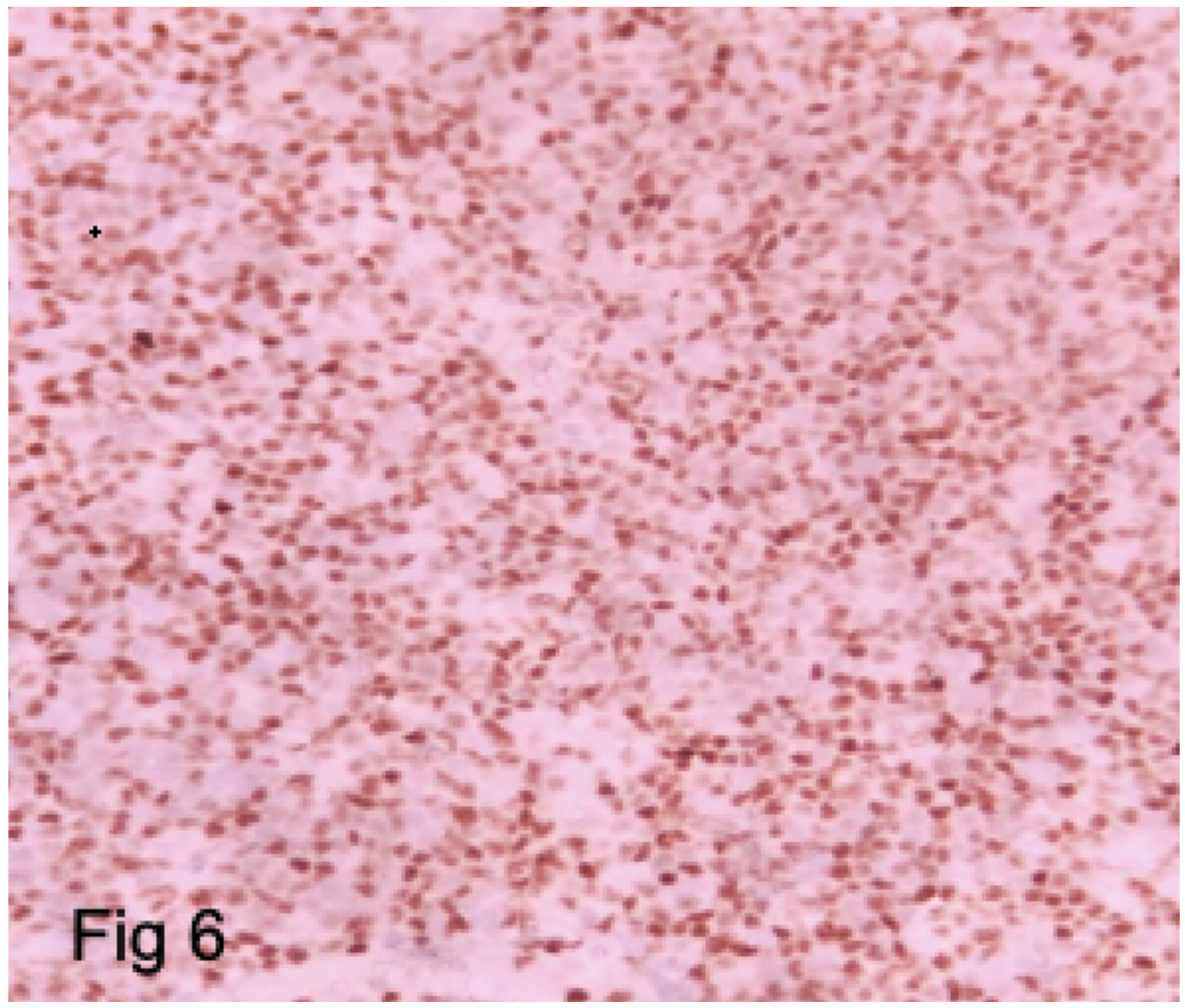

Figure 6

Diffuse nuclear staining for c-myc in $>40 \%$ lymphoma cells in Double expressor type DLBCL (X400) 


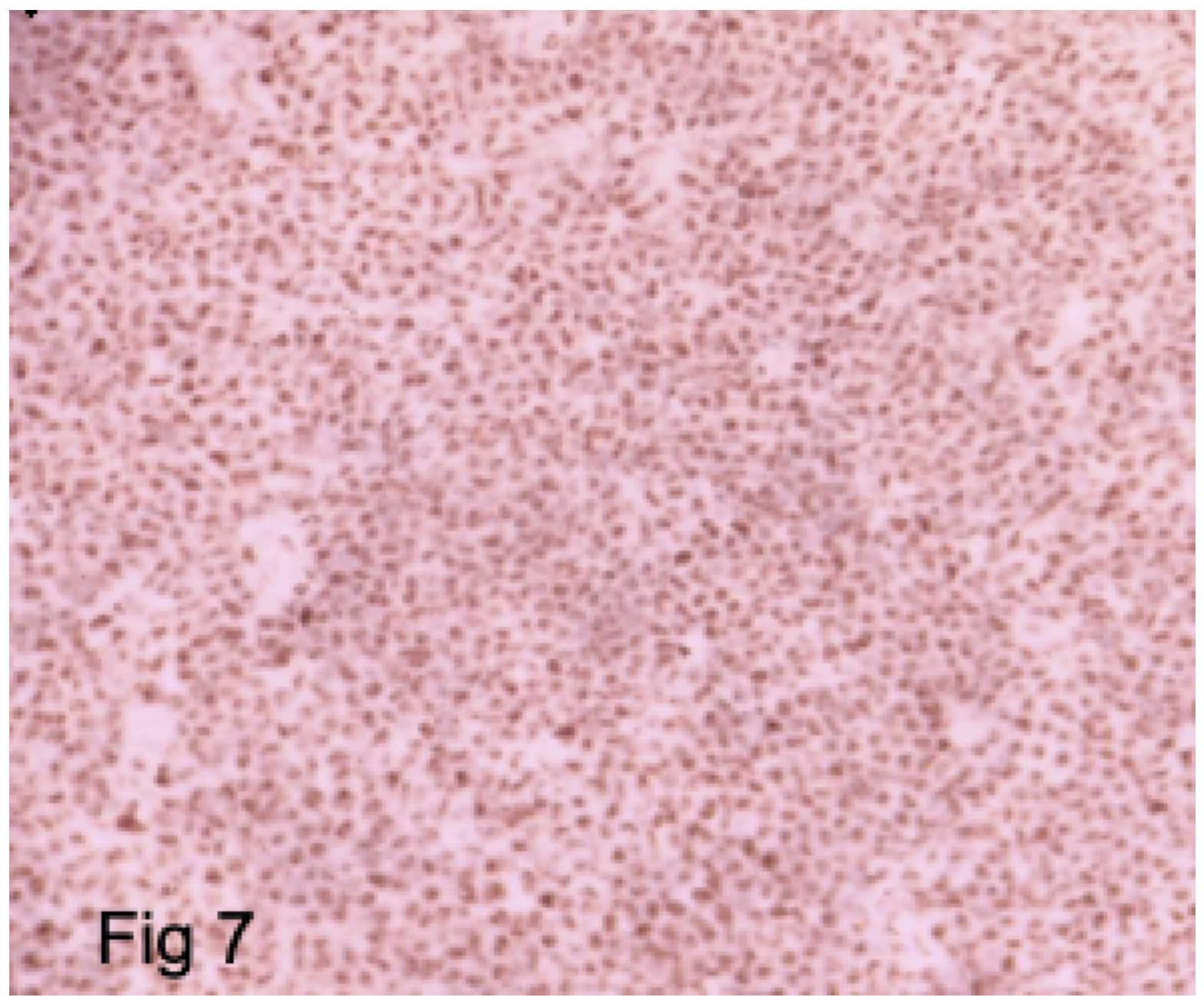

Figure 7

Diffuse nuclear staining for FOXP-1 in tumor cells in Non-GCB Type DLBCL ( X400) 


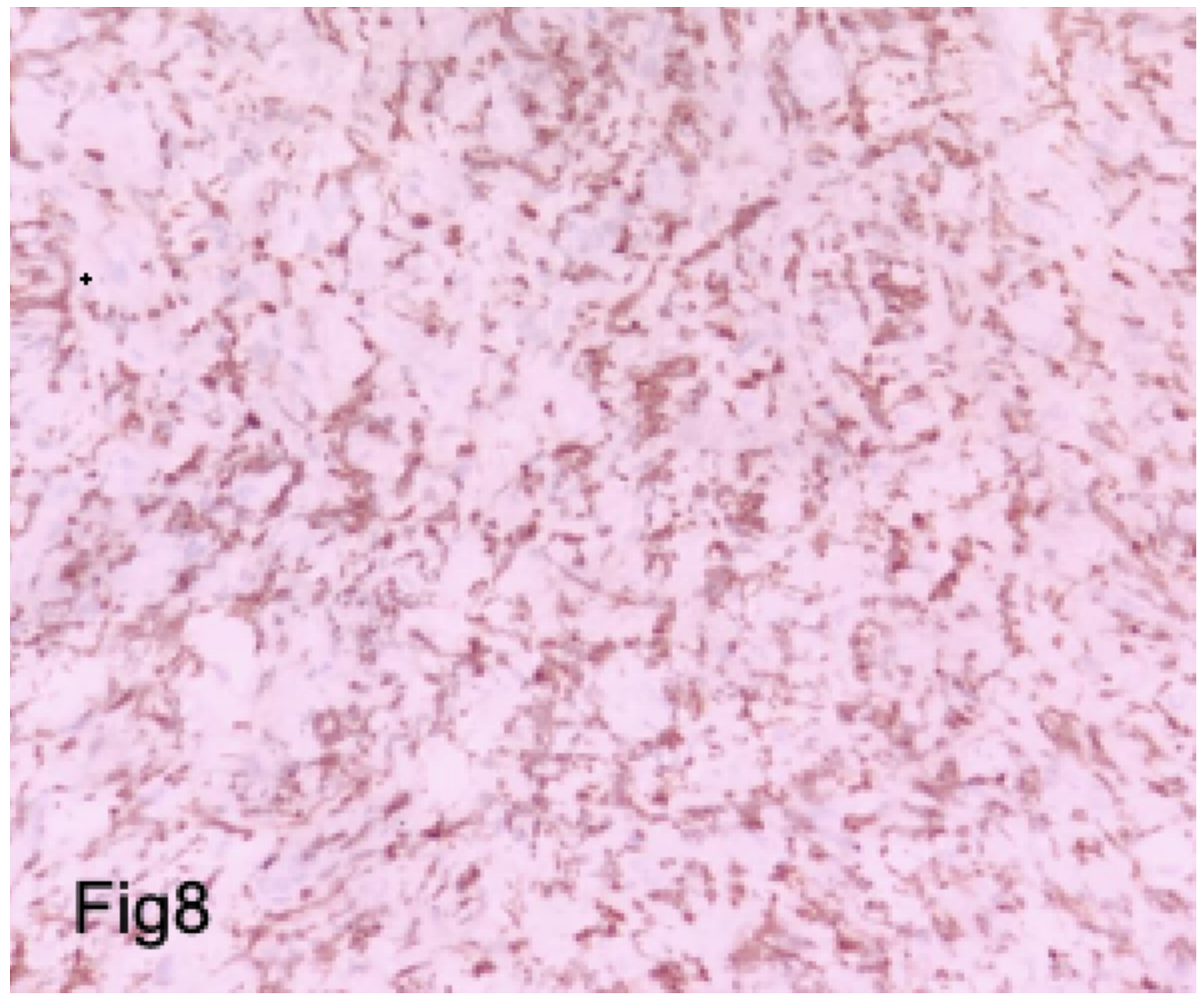

Figure 8

Nuclear staining for MUM-1 in tumor cells in Non-GCB Type DLBCL(X400) 


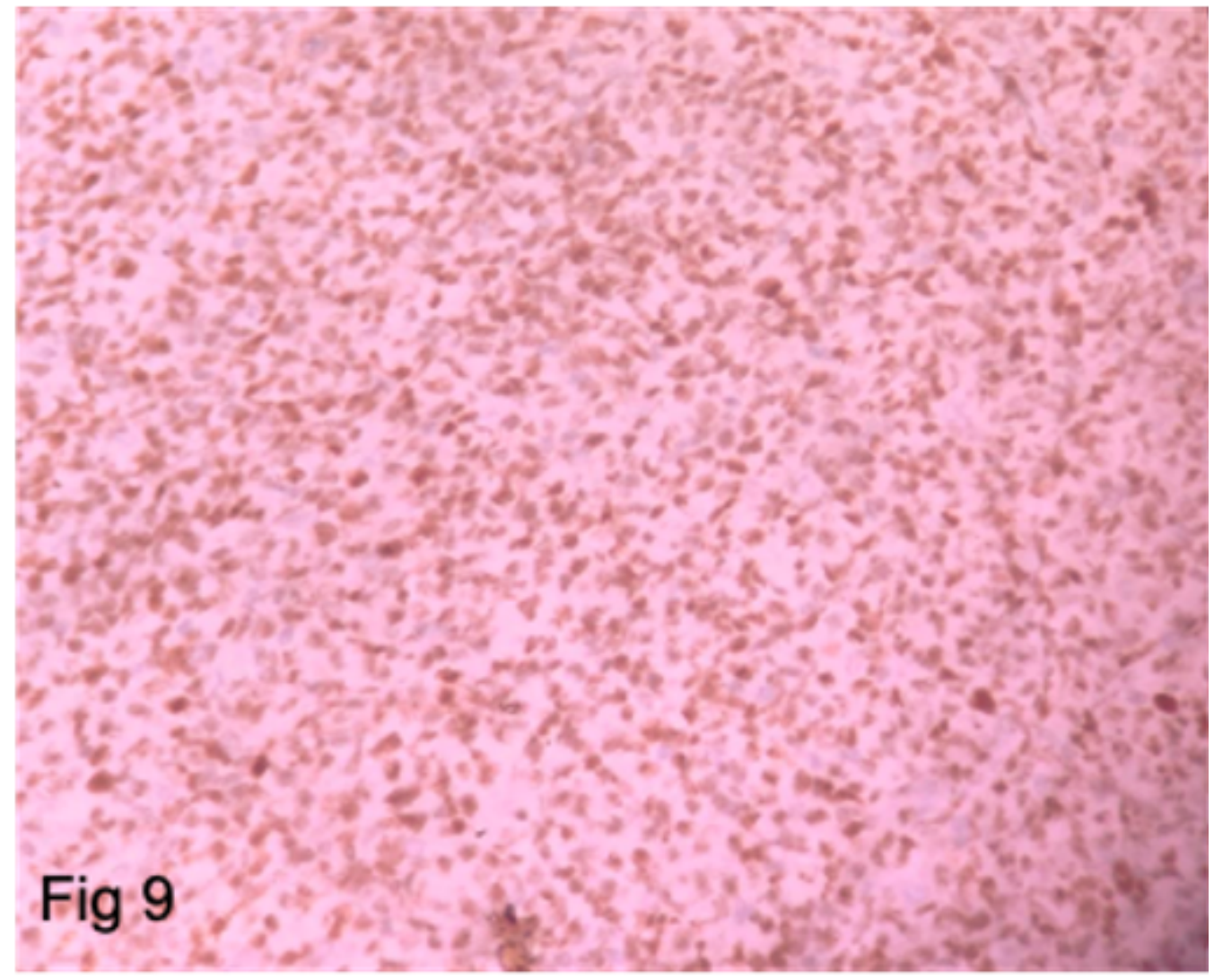

Figure 9

A.Diffuse strong nuclear staining for bcl-6 in tumor cells in GCB type DLBCL(X400)

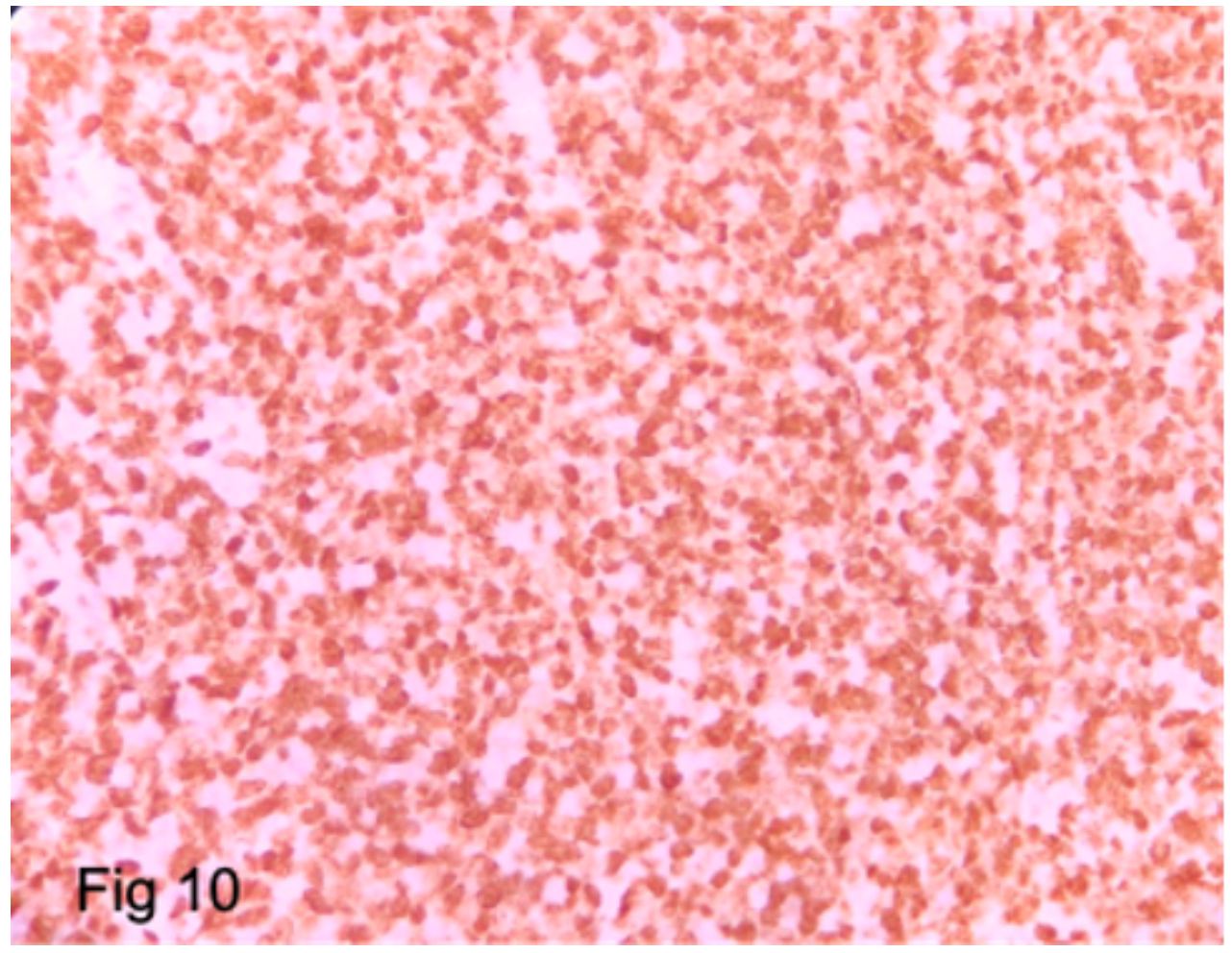

Figure 10 
High Ki-67 labelling index (80-90\%) in Lymphoma cells(x400)

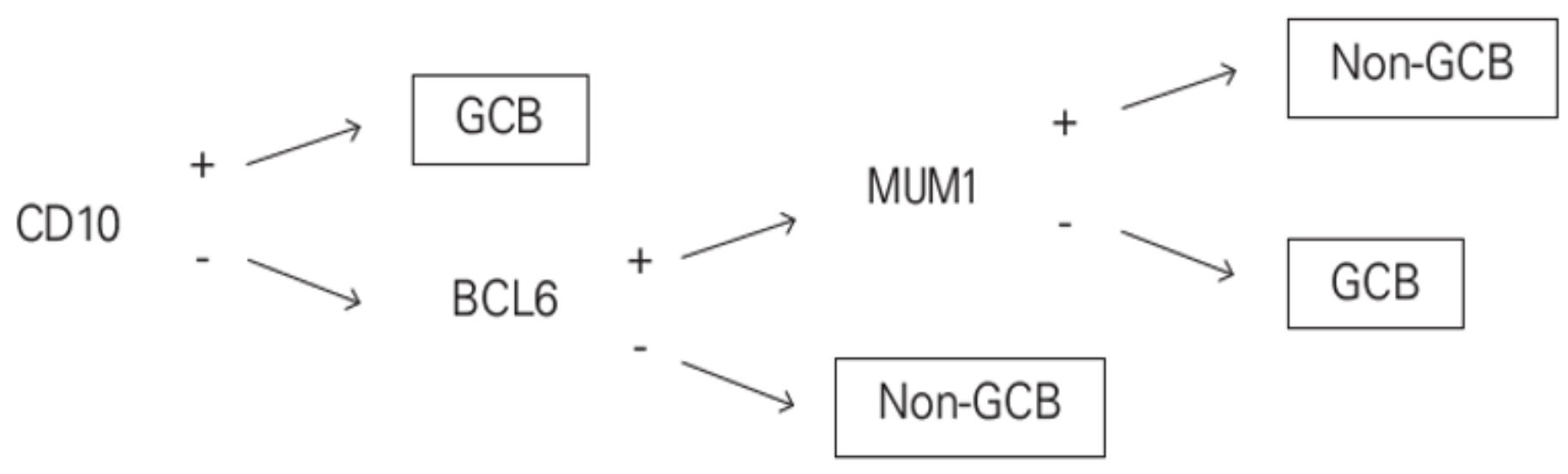

Figure 11

Hans Algorithm to approximate Molecular Subtypes Based on Immunohistochemical Patterns . GCBGerminal Centre B-cell Like 
A

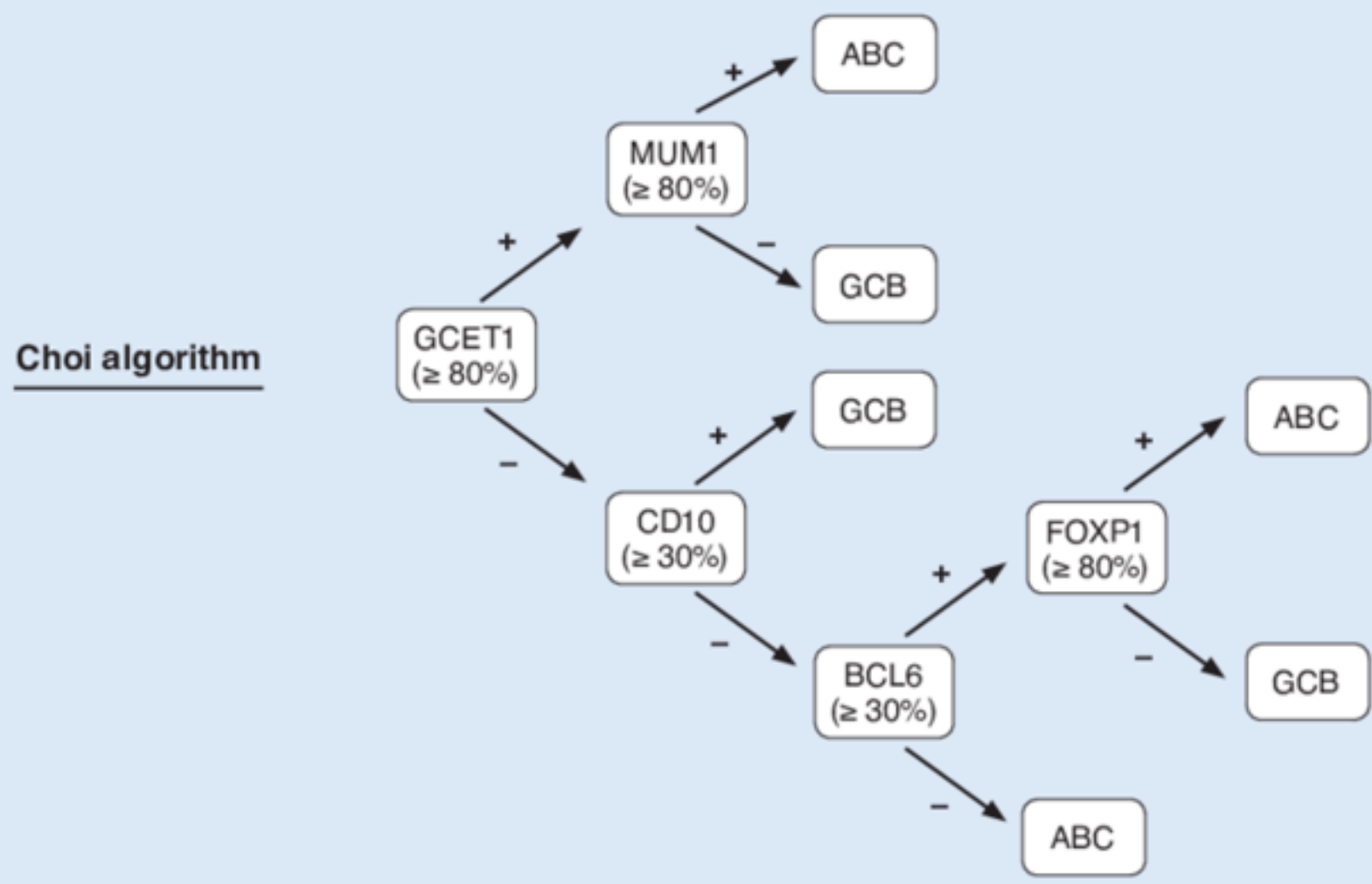

B

Tally algorithm

\begin{tabular}{|c|c|c|}
\hline $\begin{array}{c}\frac{\mathrm{GCB}}{\mathrm{CD} 10(+ \text { or }-)} \\
\operatorname{GCET} 1 \text { (+ or }-)\end{array}$ & $\begin{array}{c}\text { ABC } \\
\text { Mum1 (+ or }-) \\
\text { FoxP1 (+ or }-)\end{array}$ & $\begin{array}{c}\frac{\text { Score }}{\mathrm{GCB}}>\mathrm{ABC} \\
\text { or }\end{array}$ \\
\hline Score $(0,1,2)$ & Score $(0,1,2)$ & $\mathrm{ABC}>\mathrm{GCB}$ \\
\hline
\end{tabular}

Figure 12

Choi and Tally Algorithm to determine cell - of - origin based on munohistochemistry. 